\title{
La valuación de empresas en México Aplicación del modelo de Valor Económico Agregado: 1991-2000
}

\author{
María Luisa Saavedra García
}

\begin{abstract}
Resumen
El objetivo de este artículo es mostrar la aplicación del modelo de Valor Económico Agregado en las empresas que cotizan en la Bolsa Mexicana de Valores con el fin de determinar su valor y buscar si existe algún patrón de comportamiento del modelo al valuar a este conjunto de empresas por un período de 10 años (1991 a 2000).

En esta investigación se propuso y se aplicó una metodología para la determinación del valor en empresas mexicanas que cotizan en la Bolsa Mexicana de Valores con el modelo de Valor Económico Agregado, considerando que sólo se contaba con información pública de este conjunto de empresas.

El principal hallazgo de esta investigación es que el modelo de Valor Económico Agregado sí sigue una tendencia consistente al valuar las empresas que cotizan en la Bolsa Mexicana de Valores; si se toma como patrón de referencia el precio de mercado, se podría decir que de manera general este modelo subestima el valor de estas empresas; esta subestimación se explicó por las variables vinculadas al mismo modelo sin considerar otros aspectos.
\end{abstract}

Palabras Clave: Valor Económico Agregado, costo de capital, precio de mercado, valuación de empresas, Bolsa Mexicana de Valores.

\section{Introducción}

Entre los principales modelos de valuación de empresas ${ }^{1}$ surge el modelo de Valor Económico Agregado (EVA) ${ }^{2}$, que determina el valor de la empresa por su capacidad de ganar una tasa de rendimiento superior a su costo de capital, así como la habilidad de la administración para diseñar estrategias ${ }^{3}$ dirigidas hacia la creación de valor ${ }^{4}$.

Sin embargo, no se le ha dado la difusión y uso correcto debido a la complejidad de su aplicación y a la dificultad para entender su lógica. Así, lo que se pretende en esta investigación es demostrar la viabilidad de su aplicación en las empresas que cotizan en la

\footnotetext{
Profesora Investigadora de la Universidad Autónoma del Estado de Hidalgo. Tutora del Programa de Posgrado en Ciencias de la Administración de la Universidad Nacional Autónoma de México.

Correo electrónico: maluisasaavedra@yahoo.com

${ }^{1}$ Según la American Management Association (2000, p. 3), los pioneros de la valuación de empresas son G. Bennett Stewart III y Joel Stern, iniciadores del concepto de Valor Económico Agregado (EVA).

${ }^{2}$ EVA por sus siglas en inglés (Economic Value Added). EVA registro de patente norteamericano propiedad de Stern Stewart Co.

${ }^{3}$ Hay quienes han analizado la incorporación del EVA a las estrategias corporativas, entre otros véanse: Biddle (1997), Chen (2001), Pettit (2000), Pressly (1999), Prober (2000) y Stewart (2001).

${ }^{4}$ Varios autores han analizado el EVA y su aplicación en las empresas; también han realizado comparaciones con otros modelos. Entre otros véase: Bowen (1999), Chen (2001), Dodd y Chen (1997), Grundy (1998), Koller (1994), Milla (1998), Preusche (2000), Cleverley (2000) y Trasviña (2000).
} 
Bolsa Mexicana de Valores y que los resultados de esta aplicación son congruentes con la teoría financiera y con las pruebas empíricas realizadas por otros investigadores.

En este trabajo las preguntas de investigación se centran en lo siguiente: ¿De qué manera el modelo de valuación ${ }^{5}$ de empresas de Valor Económico Agregado, aplicado a las empresas que cotizan en la Bolsa Mexicana de Valores, sobrestima o subestima su valor? ¿La subestimación o sobrestimación se explica por variables vinculadas al modelo, o quizá por otros aspectos ${ }^{6}$ ?

El objetivo de la investigación es determinar cómo se valúa a las empresas que cotizan en la Bolsa Mexicana de Valores aplicando el modelo de Valor Económico Agregado para determinar si hay subestimación o sobrestimación del valor y si la misma se explica por variables vinculadas al modelo o quizá por otros aspectos.

\section{Modelo de Valor Económico Agregado}

El Valor Económico Agregado (EVA, por sus siglas en inglés) se define como las utilidades en operación menos el costo de capital empleado para generar esas utilidades (American Management Association, 2000: 56). El EVA se incrementará si las utilidades en operación aumentan, siempre y cuando no se haya involucrado capital adicional.

El EVA se incrementará cuando el capital fresco se invierta en proyectos con ganancias superiores al costo total del capital o si el capital pueda ser reorientado o extraído de áreas de negocios que no proporcionan un retorno aceptable; por el contrario, disminuirá cuando la administración dirija fondos a financiar proyectos con ganancias menores al costo de capital o pase por alto el fondeo de proyectos con ganancias aparentemente mayores al mismo.

Los componentes básicos del EVA son la utilidad de operación, el capital invertido y el costo de capital promedio ponderado.

- La utilidad de operación. La utilidad de operación que se usa para el cálculo del EVA es la utilidad operativa después de impuestos (UODI), que incluye los ingresos de operación, sin intereses ganados, dividendos y otros ingresos extraordinarios; los gastos incurridos en la operación de la empresa, incluyendo depreciaciones e impuestos, sin tomar en cuenta intereses a cargo u otros gastos extraordinarios. Es importante eliminar la depreciación de la utilidad operativa.

- El capital invertido. Son los activos fijos más el capital de trabajo operativo y otros activos; otra forma de llegar al capital invertido es mediante la deuda de corto y largo

\footnotetext{
${ }^{5}$ En el contexto de la presente investigación no se considera la teoría del valor, sino sólo el valor muy precisado por una minimización financiera; es decir, el valor se reduce a términos financieros. En este sentido Van Horne (1993: 102) señala "el valor para la empresa se crea mediante las inversiones de capital que aprovechan las oportunidades de rendimientos excedentes, aquéllas que proporcionan rendimientos en exceso con respecto a los que los mercados financieros requieren para el riesgo supuesto". Por otra parte, Rappaport (1986: 81) indica que las vías para crear valor son el atractivo de la industria y la ventaja competitiva. Por último, Taggart et al. (1994: 69) señalan que los determinantes financieros de la creación de valor son el rendimiento sobre el capital, la tasa de crecimiento del capital y el costo de capital.

${ }^{6}$ Los otros aspectos podrían ser las características del sector, el comportamiento de las variables del entorno económico, etc., que no serán desarrollados en la presente investigación.
} 
plazo con costo más el capital contable. El capital de trabajo operativo no toma en cuenta los pasivos con costo ni pasivos diferidos de impuestos a corto plazo.

- El costo de capital promedio ponderado. Éste se obtiene de dos fuentes: deuda con acreedores, sujeta a intereses, y el capital de los accionistas. Tanto el promedio ponderado del costo de la deuda después de impuestos como el costo del capital propio conforman el costo de capital promedio ponderado.

Es decir, el EVA es la diferencia entre la utilidad que la empresa obtiene con sus operaciones y el cargo de capital proveniente de los inversionistas.

\subsection{Métodos para calcular el EVA}

Los métodos para calcular el EVA son:

\subsubsection{Método Spread}

- El spread entre la tasa de retorno del capital y el costo de capital promedio ponderado

- Multiplicar el spread por el capital invertido

$$
\text { EVA }=(\mathbf{r}-\text { CCPP }) \times \text { Capital invertido }
$$

Donde:

$\mathbf{r}$

\section{CCPP}

Capital invertido
$=$ Tasa de rendimiento sobre el capital invertido

= Costo de capital promedio ponderado

$=$ Capital invertido al inicio

La fórmula para obtener la tasa de retorno del capital invertido es:

$$
\mathbf{r}=\quad \text { Flujo de caja disponible }
$$

Capital invertido 


\subsubsection{Método Residual}

- Obtener la utilidad operativa neta después de impuestos (UODI)

- Restar a la UODI el cargo por el uso de capital

$$
\text { EVA = UODI - }(\text { Capital invertido } \times \text { CCPP })
$$

UODI

\section{Capital invertido} CCPP
= Utilidad operativa después de impuestos

$=$ Capital invertido al inicio

$=$ Costo de capital promedio ponderado

\subsection{Estrategias del EVA}

Las estrategias que se deben aplicar con el fin de incrementar el EVA son:

- Mejorar las utilidades operativas sin mantener más capital en la empresa.

- Disponer de más capital como si fuera una línea de crédito mientras las utilidades adicionales perciban beneficios en proporción mayor al cargo que por el uso de capital se recibiría.

- Liberar capital para disminuir el nivel de crédito utilizado, en tanto que las utilidades que se van a perder sean menores a los cargos que por uso de capital se dejarán de recibir.

\subsection{Valuación de la empresa mediante la Eva}

De acuerdo con lo anterior, el EVA es la medición del desempeño financiero basado en la utilidad operativa después de impuestos, en la inversión en activos requeridos para generar esta utilidad y en el costo de la inversión en activos o el costo de capital promedio ponderado (Brewer, 1999: 4).

Para obtener el valor de una empresa mediante el EVA, debemos tomar en cuenta cuatro factores que se encuentran bajo la dirección y el control de la gerencia:

- UODI, la utilidad operativa después de impuestos esperada por el uso de esos activos antes de costos financieros y otros conceptos que no implica movimientos de efectivo.

- El beneficio fiscal de la deuda asociado con la estructura de capital objetivo.

- Cantidad del capital fresco invertido para alcanzar el crecimiento en un año normal del ciclo de inversión.

- La tasa de retorno después de impuestos esperada de las nuevas inversiones de capital. 
De acuerdo con esto el valor de la empresa será:

\section{Cuadro No 1}

\begin{tabular}{|c|c|}
\hline \multicolumn{2}{|c|}{$\begin{array}{c}\text { Valor intrínseco de la Empresa } \\
=\end{array}$} \\
\hline $\begin{array}{c}\text { Valor presente de los flujos de efectivo } \\
\text { disponible }\end{array}$ & $\begin{array}{c}\text { Menos: Valor presente de la deuda con } \\
\text { costo }\end{array}$ \\
\hline
\end{tabular}

Fuente: Valuation. Copeland (1994: 169)

El modelo, con la finalidad de incrementar el valor, requiere de la gerencia:

- Incrementar el nivel de utilidades derivadas de la operación normal del negocio.

- Crear una estructura de capital objetivo que emplee proporciones de deuda orientadas a la optimización del EVA.

- Identificar formas para incrementar las inversiones de capital en negocios donde puedan generarse retornos atractivos.

- Retirar capital de segmentos del negocio en donde se perciben tasas de retorno inadecuadas.

Los factores que no controlados por la gerencia son:

- El costo de capital por riesgo del negocio se define como es el retorno requerido por los inversionistas para compensar los riesgos de variación en los pronósticos de la utilidad operativa después de impuestos. Al combinarlo con el costo de la deuda (menos beneficio fiscal) se convierte en costo de capital promedio ponderado.

- El período futuro, en años, al final del cual los inversionistas esperan que la gerencia tendrá oportunidades de inversión atractivas. Generalmente, el avance lo fijan por las fuerzas competitivas del mercado, del desarrollo tecnológico imprevisible y las limitaciones de tamaño.

\subsection{EI EVA en las unidades de negocio}

Una adecuada valuación puede mostrar cuáles son las unidades de negocio que están creando valor y cuáles no, qué unidades son candidatas a ser vendidas y quiénes necesitan ser reestructuradas. La valuación también puede emplearse para aislar ventajas competitivas o debilidades comparativas dentro de una unidad integrada de negocio; de esta forma se recortará la distribución de recursos ya sea para capitalizar con base en fuerzas o rectificar o desprenderse con base en debilidades.

Un marco de valuación puede educar al personal operativa en lo que ellos realmente deben lograr para incrementar el valor de la unidad. Proporcionar a los gerentes educación sobre los fundamentos de valuación es clave para agilizar la toma de decisiones y facilita la comunicación a través de toda la empresa. 


\subsection{Conductores del EVA}

Ventas; costo de ventas, gastos de operación e impuestos; cargo por capital invertido son los conductores del EVA (ver gráfico 1). A continuación revisaremos en qué consisten:

- Ventas. La combinación de productos, unidades vendidas y margen de utilidad, determinan las ventas totales. La estrategia para vender productos, que generen alto valor económico agregado (EVA) con amplios márgenes de utilidad y colocar el mayor número de unidades, debe ser labor de la dirección en su conjunto.

- Costo de ventas, gastos de operación e impuestos. Elaborar estrategias de compras, inventarios, gastos de propaganda, gastos administrativos, gastos de investigación, gastos de mercadotecnia y otros gastos operativos para eficientar su uso, así como generar un vasto margen operativo. Los impuestos se calculan sobre la utilidad operativa.

- Cargo por capital invertido. Se denomina capital invertido a la inversión en inmuebles, maquinaria y equipo más el capital de trabajo operativo (sin considerar el efectivo y las inversiones temporales, así como el pasivo con costo) y otros activos. El cargo por capital invertido es el capital invertido por la tasa del costo de capital promedio ponderado.

\section{Gráfico No 1}

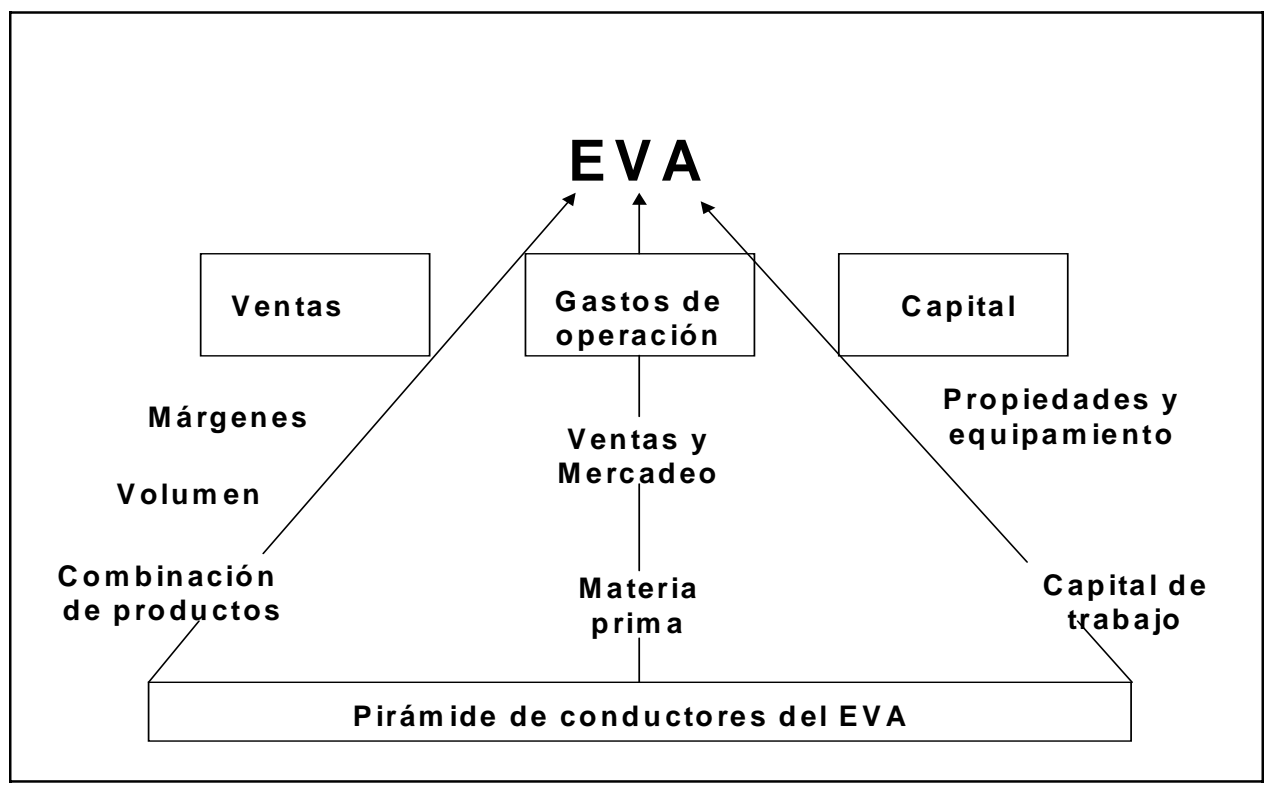

Fuente: American Management Association (2000: 52) 
El proceso del EVA debe iniciarse en el nivel de la alta gerencia y de las finanzas, utilizándolo diariamente e incorporándolos en los procesos de planeación y presupuestos. A medida que el proceso se vuelve más claro, debe ser distribuido hacia abajo a través de las líneas de autoridad de la organización. Algunas empresas cometen el error de tratar de implementar el EVA en toda su amplitud de una sola vez; debe revisarse los recursos, políticas y procedimientos establecidos que se utilizarán para implementar y controlar el proceso una vez que esté listo. Cuando las políticas y procedimientos estén terminados, la alta gerencia solicitará la conclusión de estos cambios de políticas, y cada departamento será requerido para proporcionar los criterios aplicables a su área para asegurar su aceptación.

El EVA se basa en flujos de caja y no en utilidades, al descontar el EVA generado por un proyecto determinado, automáticamente éste equivaldrá al valor presente neto ${ }^{7}$. Por lo tanto, se acepta el proyecto que produzca un EVA descontado positivo.

Si el EVA de una empresa se prevé que sea positivo significa que la empresa generará un valor superior a los costos de usar el capital que se invertirá; si el EVA se prevé que sea negativo significa que no se generará valor agregado. La medición del EVA es un indicativo respecto a cómo puede reaccionar el mercado ante una organización. Un EVA positivo puede dar lugar a una alza en el valor de mercado de las acciones o ser eventualmente tomado para ser descontado. Ésta es una de las grandes fuerzas del EVA, que además es una cualidad que lo coloca por encima de otros indicadores financieros sobre desempeño, incluyendo el flujo de caja.

El EVA es un indicador aceptable tanto de valor como de desempeño, pues relaciona la valuación estratégica a futuro, los pronósticos de inversiones de capital y los procesos presupuestales. También indica dónde establecer objetivos y metas, evaluar desempeños, fijar bonos, información importante para los inversionistas y para la preparación de pronósticos de inversiones de capital y valuaciones diversas. Sobre esta base debe erigirse el nuevo sistema de administración financiera.

\subsection{Ventajas y desventajas del EVA}

\section{Ventajas:}

- Provee una medición para la creación de riqueza que alinea las metas de los administradores de las divisiones o plantas con las metas de la compañía.

- Permite determinar si las inversiones de capital están generando un rendimiento mayor a su costo.

- Permite identificar a los generadores de valor en la empresa.

\footnotetext{
${ }^{7}$ El costo del nuevo capital empleado para financiar un proyecto es específicamente sustraído en el cálculo del EVA.
} 
- Los administradores que son compensados en función del EVA toman acciones consistentes con la generación de valor (Biddle, 1997: 301).

- Combina el desempeño operativo con el financiero en un reporte integrado que permite tomar decisiones adecuadas.

\section{Desventajas:}

- El EVA no es comparable cuando existen diferencias en los tamaños de las plantas o divisiones; por ejemplo, una gran planta o división puede tener un EVA alto y una pequeña planta un EVA bajo.

- El EVA es un cálculo que depende de los métodos de la contabilidad financiera para la realización de los ingresos y el reconocimiento de los gastos. Esto puede motivar a los administradores a manipular estos números; por ejemplo, pueden manipular el reconocimiento de los ingresos durante un período eligiendo qué órdenes de clientes despachar y cuáles retrasar. Las órdenes más rentables pueden ser aceleradas al final del período contable y enviadas al cliente antes de la fecha acordada (Pressly, 1999: 36).

- El EVA sobreenfatiza la necesidad de generar resultados inmediatos; por lo tanto, esto desincentiva a los administradores para invertir en productos innovadores o procesos tecnológicos que generan resultados en el largo plazo.

El EVA es un modelo de valuación de empresas que actualmente ya lo usan empresas como Coca Cola y Wall Mart (Pressly, 1999: 37); sin embargo la mayoría de las empresas en México aún no lo aplican. Indudablemente, es fundamental que este modelo se difunda más y que cada vez más empresas aprendan a utilizarlo como una herramienta para medir la eficiencia tanto operativa como financiera.

\section{Metodología para el cálculo del modelo de Valor Económico Agregado (EVA) para determinar la generación de valor}

\subsection{Variables del modelo:}

De acuerdo con la metodología recomendada para México por Stern Stewart ${ }^{8}$ y Co., las variables de este modelo son:

a. NOPAT = Utilidad de Operación después de impuestos

b. $\mathrm{C}^{*} \quad=$ Costo de Capital promedio ponderado de la compañía

c. Capital = Monto de la inversión para producir el NOPAT

Para determinar el EVA por el método de residual se aplica la siguiente fórmula:

$$
\mathrm{EVA}=\mathrm{NOPAT}-\left(\mathrm{C}^{*} \mathrm{x} \text { Capital }\right)
$$

\footnotetext{
${ }^{8}$ Citado en: Livas Raúl (2000; p. 2).
} 


\subsection{Medición de las variables:}

Para calcular el valor de las empresas que cotizan en la Bolsa Mexicana de Valores, aplicando el EVA, las variables se medirán como sigue:

a. NOPAT $=$ Utilidad de operación menos impuestos

Indicador : miles de pesos

b. $\mathrm{C}^{*}=$ Costo de capital promedio ponderado (CCPP)

Indicador : porcentaje

El primer elemento del CCPP es el costo del capital propio, el modelo más usado para determinar este costo es el modelo de CAPM (Capital Asset Pricing Model). Analizando la información histórica del Mercado de Valores Mexicano para el período 1991-2000, se observó que los rendimientos del mismo en algunos años de la serie histórica son negativos; esto hace que no sea posible usar el Modelo de CAPM para el cálculo del costo del capital propio.

Por lo anterior, se aplicará el método de la prima de riesgo, que de acuerdo con Haime (1998: 152) consiste en aplicar una prima por riesgo al costo de la deuda de la empresa. Esta prima de riesgo se puede determinar por el diferencial existente entre el rendimiento del mercado y la tasa libre de riesgo. Sin embargo, para México, calcular así la prima de riesgo no es aplicable porque el mercado de valores es sumamente volátil y endeble, además como puede tener rendimientos anualizados de más de 120\% (año1991), puede dar resultados negativos en los rendimientos anualizados (años 94, 98 y 2000); por lo anterior, calcular de esa forma este diferencial nos puede llevar a resultados erróneos. Así, por ejemplo, tenemos que para el año 1999 el rendimiento del mercado fue $80.1 \%$, el rendimiento de los Cetes a 28 días fue $21.41 \%$, la prima de riesgo estaría del orden de $58.59 \%$, riesgo excesivo y fuera de la realidad.

Una probable solución para la problemática planteada anteriormente, según Haime (1998: 153), es calcular la diferencia entre el promedio del rendimiento de las obligaciones de las empresas del ramo contra el rendimiento de los Cetes. Sin embargo, para ser más específicos y evitar sesgos en esta investigación se está planteando calcular el riesgo de cada empresa en particular, lo que implica calcular la prima de riesgo que le corresponde a cada una de ellas. Para alcanzar este objetivo se hará el siguiente cálculo:

- $\quad$ Costo de deuda de la empresa - Tasa libre de riesgo ${ }^{9}=$ Prima de riesgo de la empresa

El costo del capital propio aplicando este método sería como sigue:

- $\quad$ Costo de capital propio = Costo de la deuda de la empresa + Prima de riesgo de la empresa

\footnotetext{
${ }^{9}$ Para este efecto se considerará la tasa de Cetes anualizada a 28 días.
} 
El segundo elemento para el cálculo del costo de capital promedio ponderado es el costo de la deuda de la empresa, el cual para efectos de esta investigación se determinará así:

$$
\text { Costo de la deuda }=\text { Intereses } / \text { Pasivo con costo }
$$

Este cálculo se hará así, en razón de que los únicos datos con que contamos son los que proporcionan los estados financieros publicados en la Bolsa Mexicana de Valores. Se decidió entonces usar la fórmula mostrada por Weston (1994: 594) para el cálculo de la tasa anual efectiva simple:

$$
\text { Tasa anual efectiva simple }=\frac{\text { Interés }}{------------}
$$

A este resultado se le hará el ajuste fiscal ${ }^{10}=$ Costo de la deuda $(1-\mathrm{T})$.

Donde $\mathrm{T}$ es la tasa fiscal del Impuesto sobre la renta a la que está sujeta la empresa, en el caso mexicano $35 \%$.

Se ponderará el costo de cada componente de acuerdo con la proporción de los mismos dentro de la estructura de capital, multiplicando cada uno por su costo ya calculado, y se sumarán estos productos para obtener el costo de capital promedio ponderado.

\section{c. Capital $=$ Capital Invertido \\ Indicador: miles de pesos}

Se calculará sumando el Activo Fijo más el Capital de Trabajo Neto, entendiéndose este último como el activo circulante menos el pasivo circulante sin costo.

\section{Determinación de la muestra}

La muestra está conformada por 71 empresas que cotizan en la Bolsa Mexicana de Valores, de éstas se pudieron obtener los datos en las bases de datos de la propia BMV y de la Comisión Nacional Bancaria y de Valores para el caso de los Bancos. Se consideró un período de estudio de 10 años (1991 a 2000) para reflejar el impacto de los cambios sexenales, los datos que se consideraron son anuales.

Las empresas se clasificaron de acuerdo con los sectores a los que pertenecen, según la clasificación usada en el medio bursátil (ver anexo 1); por ello, se decidió separar en tres partes a las empresas del sector de la industria de la transformación, atendiendo a la similitud de su operación, tenemos entonces: Alimentos, bebidas y tabaco, Industria de la transformación I e Industria de la transformación II.

\footnotetext{
${ }^{10}$ Esto en razón de que los intereses son deducibles de impuestos.
} 
Es necesario señalar que el total de empresas inscritas en la Bolsa Mexicana de Valores son 170, pero que actualmente según Yamashiro (2001: 20) sólo se encuentran operando en promedio 65 de ellas. Además, es importante saber que el IPC ${ }^{11}$, al 31 de diciembre de 2000, se encontraba integrado por 34 empresas $^{12}$, las cuales se encuentran contenidas en esta muestra.

\section{Aplicación del modelo de Valor Económico Agregado a las empresas de la muestra}

Al aplicar la metodología propuesta en el punto II se determinó el valor de las empresas que conforman la muestra para cada uno de los 10 años objeto de estudio (ver anexo 2). Los datos de las variables financieras para cada empresa se tomaron de los estados financieros publicados en el Anuario financiero de la Bolsa Mexicana de Valores.

El cálculo del valor EVA para cada una de las empresas de la muestra se presenta en el anexo No. 3, así también el cálculo del costo de capital promedio ponderado para cada una de las empresas de la muestra se presenta en el anexo No. 4, donde se puede observar que se aplicó tanto el modelo de CAPM como el de la prima de riesgo; sin embargo, al no tener datos consistentes no fue posible aplicar el primer modelo a todos los años, motivo por el cual se optó por tomar los resultados de la aplicación del método de la prima de riesgo, como ya se explicó en su oportunidad. Sólo se presentan los cálculos que corresponden al año 2000 por cuestiones de espacio.

Los valores promedio por sector, aplicando el modelo EVA, se calcularon como un promedio simple del valor de las empresas que conforman cada sector; estos valores promedio se presentan a continuación:

\begin{tabular}{|c|c|c|c|c|c|c|c|c|c|c|}
\hline \multicolumn{11}{|c|}{ Cuadro 2} \\
\hline & & & & & & & & & & \\
\hline \multicolumn{11}{|c|}{ Valor promedio sectorial del modelo EVA(1991- } \\
\hline \multicolumn{11}{|c|}{ Em̄orèsas que cotizan en la Bolsa Mexicana de } \\
\hline \multicolumn{11}{|c|}{ (Miles de pesos) } \\
\hline Sector & 1991 & 1992 & 1993 & 1994 & 1995 & 1996 & 1997 & 1998 & 1999 & 2000 \\
\hline Alimentos, bebidas y tabaco & $-350,091$ & $-1,585,139$ & $-861,228$ & $-106,201$ & $-762,659$ & $-214,743$ & $-430,859$ & $-117,675$ & 38,114 & $-164,913$ \\
\hline Comercio & $-6,429$ & $-205,670$ & $-362,409$ & $-221,238$ & $-553,489$ & $-205,180$ & $-76,830$ & $-626,481$ & $-532,861$ & $-207,915$ \\
\hline Comunicaciones y transportes & $-5,257,989$ & $-3,879,810$ & $-5,195,185$ & $-3,895,300$ & 426,687 & $-1,185,190$ & 235,174 & $1,692,387$ & 965,403 & $4,217,339$ \\
\hline Construoción & $-695,570$ & 4,048 & 178,285 & $1,088,380$ & $-1,152,170$ & $-530,494$ & 173,561 & 721,352 & 844,789 & $1,544,214$ \\
\hline Controladoras & $-1,963,031$ & $-512,238$ & $-1,021,667$ & 55,306 & $-439,642$ & 285,708 & 287,191 & $-1,193,169$ & 44,455 & $-52,621$ \\
\hline Transf. I : metálicos, minera, minerales n & 5 mettatems 31 si & berúrgation,030 & $-1,657,629$ & $-547,975$ & $-102,890$ & $-133,442$ & 155,896 & $-187,908$ & $-773,473$ & $-738,897$ \\
\hline Transf. II:celulosa y papel y quí mica & $-895,236$ & $\begin{array}{ll}13,106 \\
\end{array}$ & $-342,569$ & 119,034 & 188,674 & 135,859 & 239,989 & 264,028 & 354,245 & 306,916 \\
\hline Senvicios financieros & n.d & n.d & n.d & n.d & n.d & n.d & $-22,000$ & 267,312 & $-1,787,918$ & $-1,462,551$ \\
\hline Otros senvicios & n.d & n.d & \begin{tabular}{l|l|}
$-104,084$ \\
\end{tabular} & 8,632 & $-69,801$ & $-366,383$ & $-127,514$ & $-113,754$ & $-54,300$ & $-15,488$ \\
\hline
\end{tabular}

Los espacios donde aparece n.d. significa que no se tuvo información disponible para realizar el cálculo del valor de estas empresas.

A continuación se presenta el precio promedio de mercado para cada uno de los sectores analizados:

\footnotetext{
${ }^{11}$ Índice de Precios y Cotizaciones de la Bolsa Mexicana de Valores.

${ }^{12}$ Dato obtenido de: http://www.bmv.com.mx, Bolsa Mexicana de Valores al 31/12/2000.
} 


\begin{tabular}{|c|c|c|c|c|c|c|c|c|c|c|}
\hline \multicolumn{11}{|c|}{ Cuadro 3} \\
\hline & & & & & & & & & & \\
\hline \multicolumn{11}{|c|}{ Valor promedio sectorial del precio de mercado (1991- } \\
\hline \multicolumn{11}{|c|}{ "Émpresas que cotizan en la Bolsa Mexicana de } \\
\hline \multicolumn{11}{|c|}{ (Miles de pesos) } \\
\hline Sector & 1991 & 1992 & 1993 & 1994 & 1995 & 1996 & 1997 & 1998 & 1999 & 2000 \\
\hline Alimentos, bebidas y tabaco & $2,289,276$ & $2,721,288$ & $4,185,760$ & $3,894,291$ & $5,057,564$ & $7,337,672$ & $10,326,734$ & $11,212,536$ & $10,433,937$ & $8,661,975$ \\
\hline Comercio & $3,001,242$ & $4,607,744$ & $6,696,503$ & $7,266,213$ & $5,700,205$ & $7,524,033$ & $15,714,239$ & $8,442,143$ & $11,722,906$ & $10,578,399$ \\
\hline Comunicaciones y transportes & $24,466,516$ & $26,702,728$ & $28,811,677$ & $22,141,082$ & $34,646,566$ & $19,918,575$ & $27,806,494$ & $24,089,026$ & $58,017,632$ & $48,375,411$ \\
\hline Construcción & $8,606,090$ & $6,356,641$ & $11,661,655$ & $9,938,270$ & $11,211,363$ & $12,506,009$ & $15,747,554$ & $8,687,193$ & $17,701,119$ & $11,304,192$ \\
\hline Controladoras & $2,616,911$ & $3,260,465$ & $5,727,263$ & $6,938,556$ & $9,177,120$ & $11,317,772$ & $14,678,566$ & $9,174,496$ & $13,399,089$ & $7,036,901$ \\
\hline Transf. I : metálicos, minera, minerales $n$ & meीálib ș bs bi & terúlíł@9,494 & $2,813,822$ & $5,015,050$ & $7,359,830$ & $7,409,811$ & $10,953,165$ & $6,170,561$ & $9,341,312$ & $5,122,883$ \\
\hline Transf. Il: celulosa y papel y quí mica & $2,604,540$ & $3,309,323$ & $3,442,902$ & $6,149,485$ & $7,948,863$ & $11,371,429$ & $14,433,781$ & 10,96 & $13,728,144$ & $8,960,839$ \\
\hline Senvicios financieros & $5,551,778$ & $16,301,678$ & $14,427,807$ & $11,644,458$ & $10,942,031$ & $15,078,397$ & $20,810,714$ & $11,832,593$ & $26,431,571$ & $25,113,082$ \\
\hline Otros senvicios & n.d. & n.d. & n.d. & n.d. & n.d. & n.d. & 480,992 & 154,823 & 240,575 & $4,034,823$ \\
\hline
\end{tabular}

Tomando como patrón de referencia el precio de mercado ${ }^{13}$ de las empresas, se observa que por lo general el valor que arroja el modelo EVA resulta ser inferior al precio de mercado, por lo cual podemos decir que:

Existe una tendencia consistente en el modelo de EVA de arrojar valores menores y, por lo general, negativos que el precio de mercado, lo cual se puede interpretar como una subvaluación del valor de la empresa. Esto se debe básicamente a que este modelo considera como generador de valor el resultado de restar a la utilidad de operación neta de impuestos el cargo por el uso de capital. Una de las variables para el cálculo de este cargo por el uso de capital es el costo de capital promedio ponderado; el costo del dinero en México es muy elevado en el período objeto de estudio, por esta razón son muy pocas las empresas que logran tener un EVA positivo.

Esto último coincide con el estudio realizado por Stern Stewart ${ }^{14}$, en el que calculó el EVA para 108 empresas $^{15}$ que cotizan en la Bolsa Mexicana de Valores para el año 1999, de las cuales sólo 16 arrojaron un EVA positivo. Este resultado indica que la mayoría de las empresas (85\%) no generaron valor, sino que están destruyendo valor, puesto que no son capaces de generar utilidades suficientes para cubrir el costo del uso de capital.

\footnotetext{
${ }^{13}$ El precio de mercado se obtuvo del Anuario Bursátil de la Bolsa Mexicana de Valores. Éste se conceptualiza como el precio de la acción multiplicada por el número de acciones en circulación.

${ }^{14}$ Firma de consultoría especializada en EVA.

${ }^{15}$ Esta información se encuentra en: http://www.sternstewart_com. 2000 Stern Stewart Perfomance Ranking México.
} 
El valor EVA en los sectores: en el gráfico de tendencias del valor EVA de los sectores se puede apreciar que todos siguen la misma tendencia a través de los años; sin embargo, se puede observar que el sector Comunicaciones y Transportes sigue una tendencia más acentuada, lo cual tendría que ver con las altas inversiones con las que trabaja este sector y con la alta volatilidad que caracteriza al mismo.

\section{Gráfico No 2}

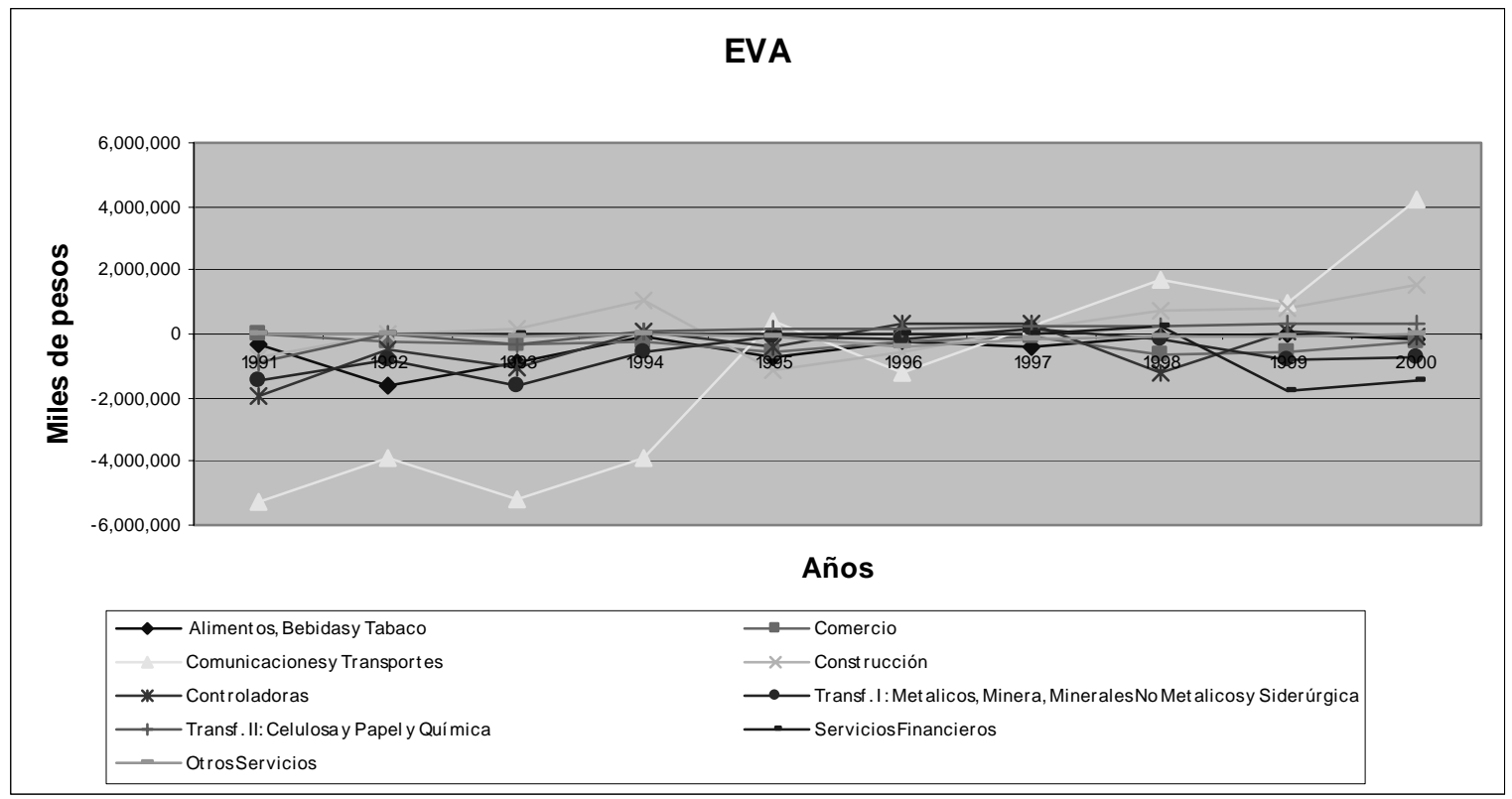

El precio de mercado en los sectores: también observamos en el gráfico de tendencia del precio de mercado que todos los sectores siguen la misma tendencia; sin embargo, nuevamente vemos que el sector Comunicaciones y Transportes sigue una tendencia más acentuada en función de ser un sector de alta volatilidad en el mercado bursátil.

\section{Gráfico No 3}

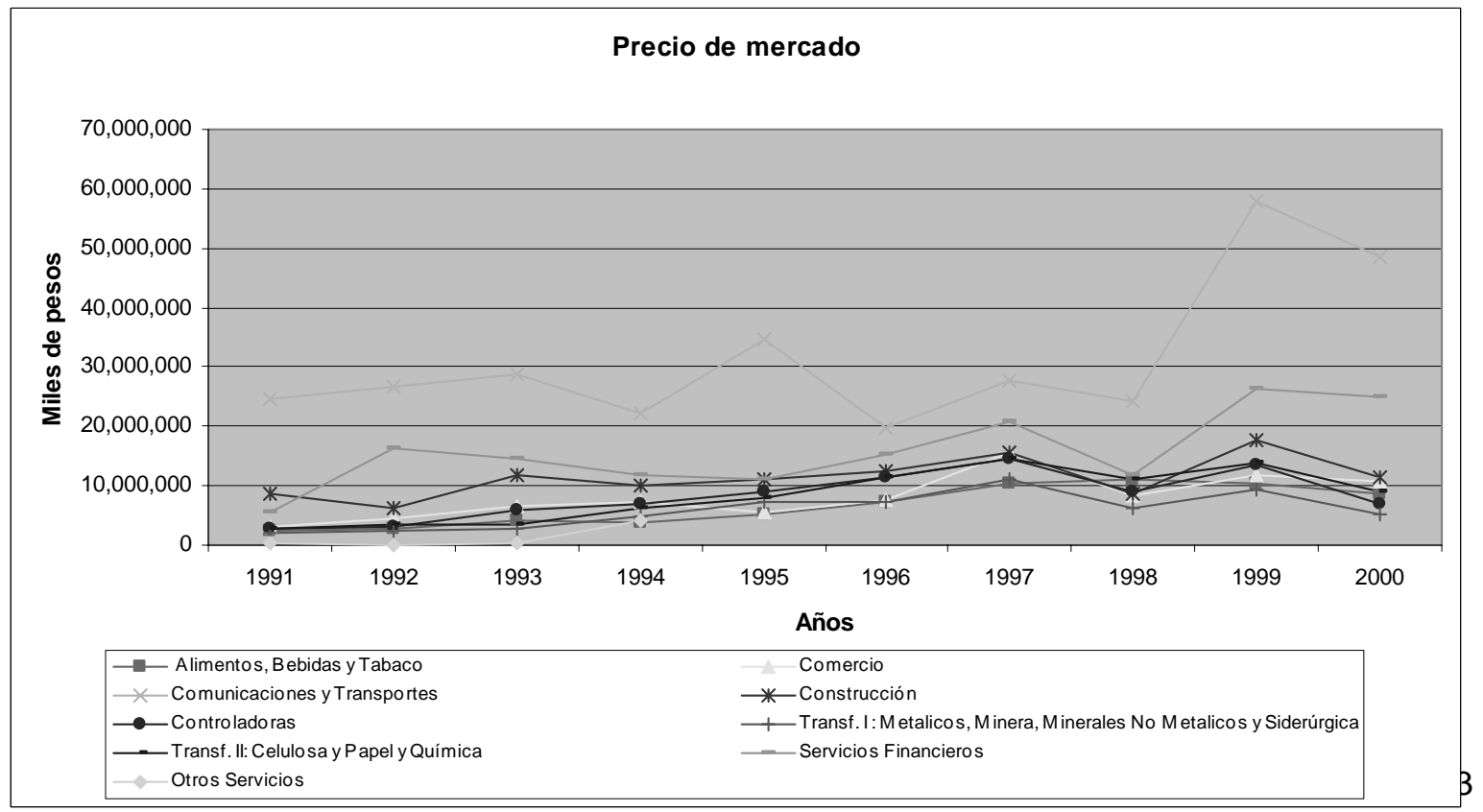


De un análisis de correlación entre los valores arrojados por el modelo EVA y el precio de mercado para cada uno de los sectores se obtuvo el siguiente resultado:

Cuadro no 4

\begin{tabular}{|l|r|}
\hline Sector & \multirow{2}{*}{$\begin{array}{c}\text { Correlación } \\
\text { EVA/ } \\
\text { Mercado }\end{array}$} \\
\cline { 1 - 1 } Alimentos, bebidas y tabaco & 0.5834 \\
\cline { 1 - 1 } Comercio & -0.0396 \\
\cline { 1 - 1 } Comunicaciones y Transportes & 0.5701 \\
\cline { 1 - 1 } Construcción & 0.1566 \\
\cline { 1 - 1 } Controladoras & 0.6739 \\
\cline { 1 - 1 } Transf. I : metal., minera, miner. no met. y siderúrgica & 0.7623 \\
\cline { 1 - 1 } Transf. II: celulosa y papel y química & 0.7414 \\
\cline { 1 - 1 } Servicios financieros & -0.8894 \\
\cline { 1 - 1 } Otros servicios & 0.7727. \\
\hline
\end{tabular}

Como observamos, una gran parte de los sectores (seis de nueve) muestran una correlación superior a $50 \%$, lo cual podría estar indicando que el EVA es un buen modelo para valuarlos correctamente sin que esto sea contundente.

Por otro lado, el sector construcción muestra una correlación débil, los sectores Comercio y Servicios financieros muestran correlaciones negativas, lo cual podría estar indicando que este modelo no es adecuado para valuar estos sectores, sin que esto sea contundente.

\section{Conclusiones}

Existe una tendencia consistente en el modelo de EVA de arrojar valores menores y, por lo general, negativos que el precio de mercado; esto se puede interpretar como una subvaluación del valor de la empresa.

Esto se debe básicamente a que este modelo considera como generador de valor el resultado de restar a la utilidad de operación neta de impuestos el cargo por el uso de capital. Una de las variables para el cálculo de este cargo por el uso de capital es el costo de capital de promedio ponderado; el costo del dinero en México es muy elevado, como ya se mencionó, para el período objeto de estudio, por esta razón son muy pocas las empresas que logran tener un EVA positivo.

Lo anterior es similar con el estudio realizado por Stern Stewart ${ }^{16}$, quien calculó el EVA para 108 empresas $^{17}$ que cotizaban en la Bolsa Mexicana de Valores en 1999; de ellas sólo 16 arrojaron un EVA positivo. Con esto se demuestra que la mayoría de las empresas

\footnotetext{
${ }^{16}$ Firma de consultoría especializada en EVA.

${ }^{17}$ Esta información se encuentra en: http://www.sternstewart.com. 2000 Stern Stewart Perfomance Ranking México
} 
(85\%) no generaron valor, sino que lo están destruyendo porque no son capaces de generar utilidades suficientes para cubrir el costo del uso de capital.

Asimismo, Preusche (2000: 12) en un análisis acerca de la generación de valor en las empresas argentinas llegó a la conclusión siguiente: uno de los problemas principales de las empresas en su esfuerzo para crear valor es el alto costo de capital, derivado del alto riesgo en el país.

El valor del EVA para cada uno de los sectores y el precio de mercado siguen la misma tendencia en cada uno de los años, mostrándose una tendencia más acentuada en el sector Transportes y comunicaciones debido a su alta volatilidad.

El resultado de la prueba de correlación entre los valores del EVA y el precio de mercado arrojo altas correlaciones en seis de los nueve sectores analizados lo que podría estar indicando que es un modelo adecuado para calcular el valor de estos sectores, sin ser esto contundente.

Los defensores del EVA han argumentado que éste es el modelo que muestra el verdadero valor, puesto que considera sólo los aspectos de la operación misma de la empresa aislando otros que pudieran distorsionar el cálculo; también indican que se debe calcular el cargo por el uso del capital no sobre las fuentes de financiamiento - como se ha hecho tradicionalmente-, sino sobre el capital invertido que se está usando para generar las utilidades de operación del negocio.

\section{Bilbiografía y Hemerografía}

American Management Association (2000), Valor Económico Agregado-EVA, México, julio.

Biddle, Gary, Bowen, Robert y James Wallace (1997), "Does EVA beat earnings? Evidence on associations with stock returns and firm values", Journal of Accounting y Economics, $\mathrm{n}^{\circ}$ 24, Estados Unidos de América, pp. 301-336.

Bolsa Mexicana de Valores (2001), Anuario bursátil de la Bolsa Mexicana de Valores, Bolsa Mexicana de Valores, México.

Bolsa Mexicana de Valores (2001), Anuario financiero de la Bolsa Mexicana de Valores, Bolsa Mexicana de Valores, México.

Bowen, Robert M. y James S. Wallace (1999), "The Decision to Adopt EVA", en Issues in Accounting Education, agosto, vol. 14, núm. 3, Estados Unidos de América, pp. 517-542.

Brewer Peter, Chandra Gyan et al. (1999), "Economic value added (EVA): Its uses and limitations S.A.M.”, en Advanced Management Journal Cincinnati, vol. 64, núm. 2, Estados Unidos de América, pp. 4-11. 
Chen, Shimin y L. James Dodd (2001), "Operating income, residual income and EVA: Which metric is more value relevant". Pittsburg : Journal of lines projected cash flow and implied growth rate". EUA : Journal of Investing; spring, pp. 38-45.

Cleverley, William (2000), "Calculating the true value of healthcare organizations", en Journal of the Healthcare Financial Management Association, Suplement 2000 HFM Resource Guide, Estados Unidos de América, pp. 4-8.

Dodd, L. James y Shimin Chen (1997), "Economic value added (EVA)", en Business and Economic Review, invierno, vol. 30, núm. 3, Arkansas, Estados Unidos de América, pp. 1-7.

Grundy, Tony (1998), "Managing the business value system", en Management Accounting; diciembre, vol. 76, núm. 11, Londres, pp. 30-32.

Haime, Luis (1998), Reestructuración integral de empresas, ISEF, México.

Horne, James van (1993), Administración Financiera, Prentice Hall, México.

Koller, Timothy (1994), "What is value-based management?", en The Mc. Kinsey Quartely, núm. 3, Estados Unidos de América, pp. 87-101.

Livas, Raúl (2000), "Eva Primer", en Mexican Market Eva Review, Stern Stewart, Co. Research, septiembre, Estados Unidos de América.

Milla, Sindy (1998), El análisis fundamental y la valuación de acciones en México, Tesis de Grado de Maestría en Finanzas, UNAM, Facultad de Contaduría y Administración, México.

Pettit, Justin (2000), "Eva and Strategy", en EVAluation, Stern Steward, Co. Research, abril, Estados Unidos de América, pp. 1-20.

Pressly, Thomas (1999), "EVA (Economic Value Added): The Real Key to Creating Wealth", en C.P.A Journal Columbus, vol. 58, núm. 4, Estados Uniidos de América, pp. 36-37.

Preusche, Alejandro, Scokin, Damián y Eduardo Urdapilleta (2000), "Value in Argentina", en The McKinsey Quarterly, núm. 3, Estados Unidos de América, pp. 11-13.

Prober, Larry (2000), "EVA: A better financial reporting tool", en Pensilvania CPA Journal, vol. 71, núm. 3, Filadelfia, Estados Unidos de América, pp. 27-33.

Rappaport, Alfred (1986), Creating Shareholder value, The Free Press, Nueva York.

Stewart, Bennett (1991), The Quest for Value, The EVA Management Guide. Harper Collins, Plubishers Inc, Estados Unidos de América. 
Stewart, Bennett (2001), "All About EVA The Real Key to Creating Wealth", en EVAluation, Stern Stewart y Co. Research, Estados Unidos de América, enero, pp. $1-16$.

Taggart, James, Kontes, Peter y Michael Mankins (1994), The Value Imperative. Managing for Superior Shareholder Returns, The Free Press, Nueva York.

Thierauf, Robert y Richard Grosse (1987), Toma de decisiones por medio de Investigación de Operaciones, Limusa, México.

Trasviña, Laura A. (2000), Valor Económico Agregado en las empresas como herramienta de análisis financiero, Tesis de Grado de Maestría en Contaduría, UNAM, Facultad de Contaduría y Administración, México.

Weston, Fred y Eugene Brigham (1994), Administración Financiera, Mc. Graw Hill, México.

Yamashiro, Celina (2001), "Las Empresas en la Bolsa Mexicana de Valores", en El Financiero: Sección de Negocios, México, 25 de junio, p.20. 
Anexo 1

Empresas que conforman la muestra

\begin{tabular}{|l|l|}
\hline No & Sector 1: Alimentos, bebidas y tabaco \\
\hline 1 & Grupo Bimbo, S.A. de C.V. \\
\hline 2 & Grupo Continental, S.A. de C.V. \\
\hline 3 & Fomento Económico Mexicano, S.A. de C.V. \\
\hline 4 & Grupo Industrial Maseca, S.A. de C.V. \\
\hline 5 & Grupo Modelo, S.A. de C.V. \\
\hline 6 & Savia, S.A. de C.V. \\
\hline 7 & Industrias Bachoco, S.A. de C.V. \\
\hline 8 & Grupo de Embotelladoras Unidas, S.A. de C.V. \\
\hline 9 & Grupo La Moderna, S.A. de C.V. \\
\hline 10 & Embotelladoras Argos, S.A. de C.V. \\
\hline 11 & Grupo Herdez, S.A. de C.V. \\
\hline 12 & Maizoro, S.A. de C.V. \\
\hline 13 & Coca Cola Femsa, S.A. de C.V. \\
\hline 14 & Gruma, S.A. de C.V. \\
\hline & \\
\hline & Sector 2: Comercio \\
\hline 15 & Controladora Comercial Mexicana, S.A. de C.V. \\
\hline 16 & Organización Soriana, S.A. de C.V. \\
\hline 17 & Grupo Elektra, S.A. de C.V. \\
\hline 18 & Wall Mart México, S.A. de C.V. \\
\hline 18 & Grupo Casa Saba, S.A. de C.V. \\
\hline 20 & Grupo Dataflux, S.A. de C.V. \\
\hline 21 & Edoardos Martín, S.A. de C.V. \\
\hline 22 & Corporativo Fragua, S.A. de C.V. \\
\hline 23 & Grupo Corvi, S.A. de C.V. \\
\hline 24 & Grupo Gigante, S.A. de C.V. \\
\hline 25 & Grupo Palacio de Hierro, S.A. de C.V. \\
\hline 26 & Alsea, S.A. de C.V. \\
\hline 27 & Nadro, S.A. de C.V. \\
\hline 28 & Puerto de Liverpool, S.A. de C.V. \\
\hline 29 & Grupo Prove-Quim. S.A. de C.V. \\
\hline & \\
\hline & Sector 3: Comunicaciones \\
\hline 30 & Carso Global Telecom, S.A. de C.V. \\
\hline 31 & Grupo Televisa, S.A. \\
\hline 32 & Teléfonos de México, S.A. de C.V. \\
\hline 33 & TV Azteca, S.A. de C.V. \\
\hline 34 & Transportación Marítima Mexicana, S.A. de C.V. \\
\hline 35 & Cintra, S.A. de C.V. \\
\hline 36 & Grupo Radio Centro, S.A. de C.V. \\
\hline 37 & Biper, S.A. de C.V. \\
\hline 38 & Grupo Iusacell, S.A. de C.V. \\
\hline & Sector 4: Construcción \\
\hline 40 & Apasco, S.A. de C.V. \\
\hline & Cemex, S.A. de C.V. \\
\hline 2 & Consorcio Ara, S.A. de C.V. \\
\hline
\end{tabular}




\begin{tabular}{|c|c|}
\hline 43 & Corporación Geo, S.A. de C.V. \\
\hline 44 & $\begin{array}{l}\text { Empresas Ica Sociedad Controladora, S.A. de } \\
\text { C.V. }\end{array}$ \\
\hline & Sector 5: Controladoras \\
\hline 45 & $\begin{array}{l}\text { Corporación Interamericana de entretenimiento, } \\
\text { S.A. de C.V. }\end{array}$ \\
\hline 46 & Alfa S.A. de C.V. \\
\hline 47 & Desc, S.A. de C.V. \\
\hline 48 & Grupo Carso, S.A. de C.V. \\
\hline 49 & Grupo Industrial Saltillo, S.A. de C.V. \\
\hline 50 & Grupo Sanbors, S.A. de C.V. \\
\hline 51 & San Luis Corporación, S.A. de C.V. \\
\hline 52 & Cydsa, S.A. de C.V. \\
\hline 53 & G. Acción, S.A. de C.V. \\
\hline 54 & Grupo Industrial Camesa, S.A. d \\
\hline & $\begin{array}{l}\text { Sector 6: Ind. transformación I: metálicos, } \\
\text { minera, minerales no metálicos y siderúrgica }\end{array}$ \\
\hline 55 & Metálicos Industrias CH, S.A. de C.V. \\
\hline 56 & Minera Grupo México, S.A. de C.V. \\
\hline 57 & Minera Industrias Peñoles, S.A. de C.V. \\
\hline 58 & Minerales no M Vitro, S.A. de C.V. \\
\hline 59 & $\begin{array}{l}\text { Siderúrgica Tubos de Acero de México, S.A. de } \\
\text { C.V. }\end{array}$ \\
\hline 60 & Siderúrgica Hylsamex, S.A. de C.V. \\
\hline & $\begin{array}{l}\text { Sector 7: Ind. transformación II: celulosa y } \\
\text { papel y química }\end{array}$ \\
\hline 61 & $\begin{array}{l}\text { Celulosa/Papel Kimberly Clark de México, } \\
\text { S.A. de C.V. }\end{array}$ \\
\hline 62 & $\begin{array}{l}\text { Celulosa/Papel Empaques Ponderosa, S.A. de } \\
\text { C.V. }\end{array}$ \\
\hline 63 & Química Tekchem S.A. de C.V. \\
\hline 64 & Química Mexichem S.A. de C.V. \\
\hline & Sector 8: Servicios financieros \\
\hline 65 & $\begin{array}{l}\text { Serv. Financieros Grupo Financiero, Banamex } \\
\text { Accival, S.A. de C.V, }\end{array}$ \\
\hline 66 & $\begin{array}{l}\text { Serv. Financieros Grupo Financiero Bital, S.A. de } \\
\text { C.V. }\end{array}$ \\
\hline 67 & $\begin{array}{lll}\text { Serv. Financieros Grupo } & \text { Financiero } & \text { BBVA } \\
\text { Bancomer, S.A. de C.V. } & & \\
\end{array}$ \\
\hline 68 & $\begin{array}{l}\text { Serv. Financieros Grupo Financiero Inbursa, S.A. } \\
\text { de C.V. }\end{array}$ \\
\hline 69 & $\begin{array}{lll}\text { Serv. Financieros Grupo } & \text { Financiero } & \text { Banorte, } \\
\text { S.A. de C.V. } & & \\
\end{array}$ \\
\hline & Sector 9: Otros servicios \\
\hline 70 & Grupo Aeroportuario del Sureste, S.A. de C.V. \\
\hline 71 & Médica Sur, S.A. de C.V. \\
\hline
\end{tabular}

Fuente : BMV (2001). Anuario Bursátil 2000. México: Bolsa Mexicana de Valores, S.A. de C.V. 
Anexo

Cálculo del valor promedio sectorial del modelo EVA (1991-2000)

Empresas que cotizan en la Bolsa Mexicana de Valores

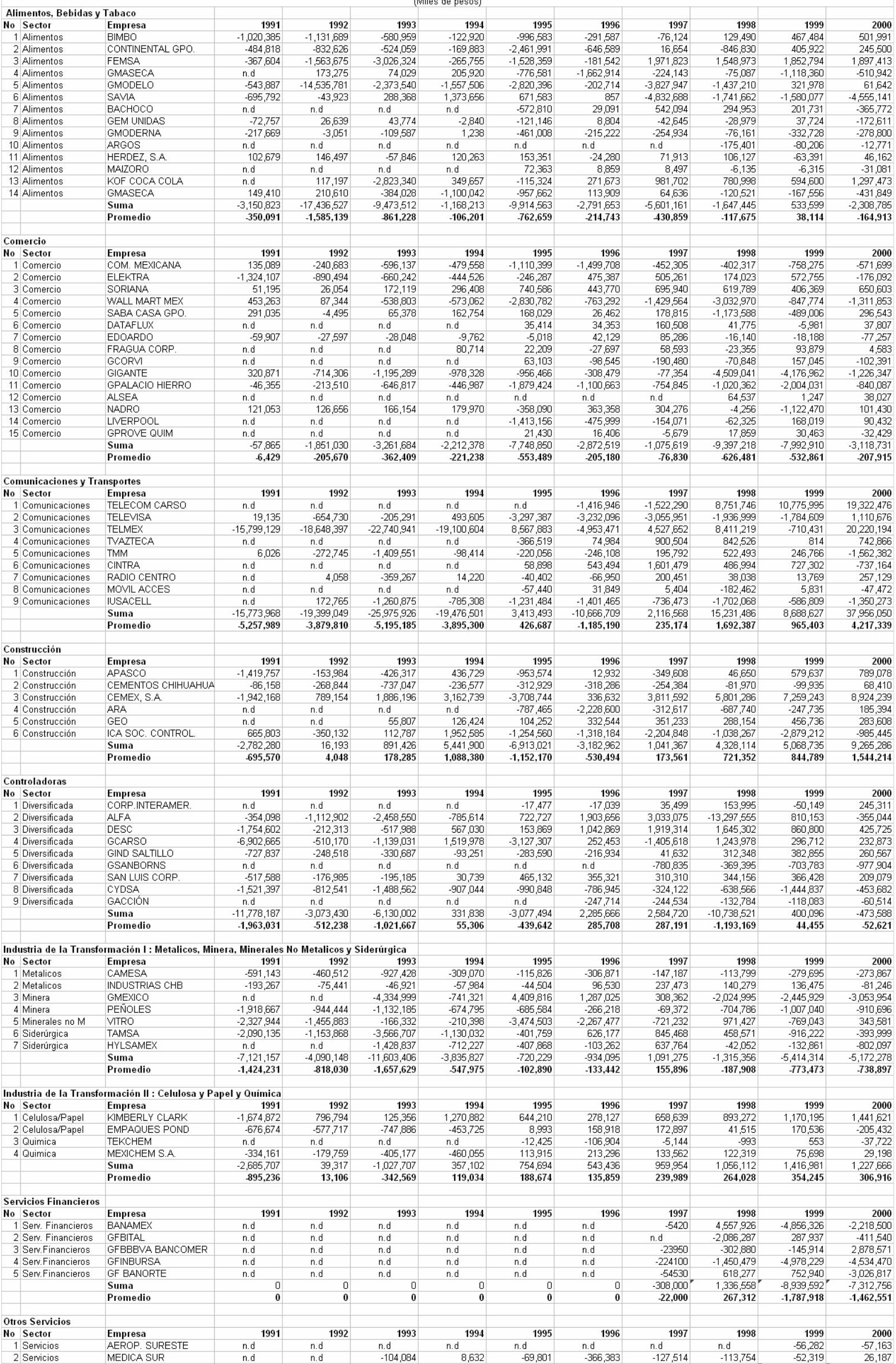




\begin{tabular}{|c|c|c|c|c|c|c|c|c|}
\hline & & & & & Anexo 3 & & & \\
\hline & & & & lores calculados & aplicando el m & delo EVA & & \\
\hline & & & & $(\mathrm{mi}$ & es de pesos) & & & \\
\hline & & & \begin{tabular}{|l|} 
Utilidad de \\
\end{tabular} & Impuesto $^{2}$ & Utilidad ${ }^{3}$ & Activ o Fij o ${ }^{4}$ & Capital de ${ }^{5}$ & Capital $^{6}$ \\
\hline EVA & año 2000 & & Operación & & Ajustada & & Trabajo & Invertido \\
\hline No & Sector & Empresa & & & & & & \\
\hline & Alimentos & BIMBO & $3,283,099$ & $1,262,466$ & $2,020,633$ & $13,572,791$ & $2,730,481$ & $16,303,272$ \\
\hline & Alimentos & CONTINENTAL GPO. & $1,782,611$ & 906,043 & 876,568 & $4,503,312$ & $1,623,561$ & $6,126,873$ \\
\hline 3 & Alimentos & FEMSA & $7,172,793$ & $2,339,422$ & $4,833,371$ & $26,866,279$ & $4,665,721$ & $31,532,000$ \\
\hline 4 & Alimentos & GMASECA & 822475 & 21312 & 801,163 & 12683732 & 1844386 & $14,528,118$ \\
\hline 5 & Alimentos & GMODELO & $7,704,075$ & $3,545,629$ & $4,158,446$ & $29,733,502$ & $10,228,997$ & $39,962,499$ \\
\hline 6 & Alimentos & SAVIA & $-2,587,114$ & 821,381 & $-3,408,495$ & $8,958,314$ & $4,036,491$ & $12,994,805$ \\
\hline 7 & Alimentos & BACHOCO & 1515867 & 398549 & $1,117,318$ & 6328078 & 2398559 & $8,726,637$ \\
\hline 8 & Alimentos & GEMUNIDAS & 276,095 & 112,029 & 164,066 & $1,394,969$ & 191,191 & $1,586,160$ \\
\hline 9 & Alimentos & GMODERNA & 273,132 & 109,838 & 163,294 & $1,400,058$ & 926,382 & $2,326,440$ \\
\hline 10 & Alimentos & ARGOS & 953,595 & 402,080 & 551,515 & $2,406,119$ & 57,198 & $2,463,317$ \\
\hline 11 & Alimentos & HERDEZ, S.A. & 534,495 & 162,443 & 372,052 & $1,608,831$ & $1,170,931$ & $2,779,762$ \\
\hline 12 & Alimentos & MAIZORO & 30,954 & 11,401 & 19,553 & 274,615 & 64,041 & 338,656 \\
\hline 13 & Alimentos & KOF COCA COLA & $2,973,403$ & 981,107 & $1,992,296$ & $7,134,677$ & $1,091,438$ & $8,226,115$ \\
\hline 14 & Alimentos & MASECA & 530,638 & 170,003 & 360,635 & $4,026,028$ & $1,953,729$ & $5,979,757$ \\
\hline 15 & Celulosa/Papel & KIMBERLY CLARK & $5,144,501$ & $2,058,519$ & $3,085,982$ & $14,074,426$ & $3,406,863$ & $17,481,289$ \\
\hline 16 & Celulosa/Papel & EMPAQUES POND & 256561 & 151280 & 105,281 & 1699331 & 680570 & $2,379,901$ \\
\hline 17 & Comercio & COM. MEXICANA & $1,120,560$ & 75,659 & $1,044,901$ & $13,773,760$ & $-181,448$ & $13,592,312$ \\
\hline 18 & Comercio & SORIANA & $1,783,906$ & $\begin{array}{ll}678,108 \\
\end{array}$ & $1,105,798$ & $12,231,307$ & 214,223 & $12,445,530$ \\
\hline 19 & Comercio & ELEKTRA & $1,676,027$ & 209,493 & $1,466,534$ & $3,716,807$ & $2,867,760$ & $6,584,567$ \\
\hline 20 & Comercio & WALL MART MEX & $3,959,205$ & $1,599,176$ & $2,360,029$ & $28,768,703$ & $6,880,641$ & $35,649,344$ \\
\hline 21 & Comercio & SABA CASA GPO. & 572,088 & 107,739 & 464,349 & 990,423 & $1,725,579$ & $2,716,002$ \\
\hline 22 & Comercio & DATAFLUX & 30,980 & $\mid-15,577$ & 46,557 & 175,187 & $-57,620$ & 117,567 \\
\hline 23 & Comercio & EDOARDO & 75,688 & 24,352 & 51,336 & 230,928 & 204,356 & 435,284 \\
\hline 24 & Comercio & FRAGUA CORP. & 210,509 & 96,834 & 113,675 & 406,562 & 580,419 & 986,981 \\
\hline 25 & Comercio & GCORVI & 258,092 & 54,299 & 203,793 & $1,003,239$ & 497,445 & $1,500,684$ \\
\hline 26 & Comercio & GIGANTE & $1,209,035$ & 356,558 & 852,477 & $12,283,092$ & $-785,170$ & $11,497,922$ \\
\hline 27 & Comercio & GPALACIO HIERRO & 496,494 & 184,034 & 312,460 & $5,334,117$ & $1,787,610$ & $7,121,727$ \\
\hline 28 & Comercio & ALSEA & 227,736 & 85,508 & 142,228 & 737,617 & 130,229 & 867,846 \\
\hline 29 & Comercio & NADRO & 494,771 & 174,010 & 320,761 & 571,356 & $2,173,187$ & $2,744,543$ \\
\hline 30 & Comercio & LIVERPOOL & $1,582,028$ & 459,789 & $1,122,239$ & $8,653,875$ & $2,485,693$ & $11,139,568$ \\
\hline 31 & Comercio & GPROVE QUIM & 25,848 & 9,701 & 16,147 & 147,744 & 21,501 & 169,245 \\
\hline 32 & Comunicaciones & TELECOM CARSO & $42,882,907$ & $14,733,300$ & $28,149,607$ & $137,548,333$ & $-23,701,085$ & $113,847,248$ \\
\hline 33 & Comunic aciones & TELEVISA & $4,897,140$ & 390,973 & $4,506,167$ & $12,728,212$ & $21,832,056$ & $34,560,268$ \\
\hline 34 & Comunic aciones & TELMEX & $40,150,267$ & $11,267,999$ & $28,882,268$ & $107,716,460$ & $-22,058,558$ & $85,657,902$ \\
\hline 35 & Comunic aciones & TVAZT ECA & $1,594,671$ & $-18,303$ & $1,612,974$ & $2,453,467$ & $5,040,896$ & $7,494,363$ \\
\hline 36 & Comunic aciones & TMM & 301496 & -86265 & 387,761 & 19031043 & 647497 & $19,678,540$ \\
\hline 37 & Comunic aciones & CINTRA & 1190848 & 378469 & 812,379 & 10180632 & -19695 & $10,160,937$ \\
\hline 38 & Comunic aciones & RADIO CENT RO & 338055 & 27147 & 310,908 & 475151 & 142396 & 617,547 \\
\hline 39 & Comunicaciones & MOVIL ACCES & -8270 & 2738 & $-11,008$ & 297224 & -149745 & 147,479 \\
\hline 40 & Comunic aciones & IUSACEШ & -410036 & 151430 & $-561,466$ & 8282348 & -659513 & $7,622,835$ \\
\hline 41 & Construcción & APASCO & $3,084,778$ & $1,156,201$ & $1,928,577$ & $10,954,873$ & 751,608 & $11,706,481$ \\
\hline 42 & Construcción & CEMENT OS CHIHUAHUA & 789,855 & 328,306 & 461,549 & $3,661,367$ & $1,129,790$ & $4,791,157$ \\
\hline 43 & Construcción & CEMEX,SA. & $16,088,758$ & $1,884,840$ & $14,203,918$ & $87,885,433$ & $-18,633,483$ & $69,251,950$ \\
\hline 44 & Construcción & ARA & 733,371 & 211,807 & 521,564 & 280,489 & $3,111,739$ & $3,392,228$ \\
\hline 45 & Construcción & GEO & 669,546 & 101,843 & 567,703 & 781,725 & $2,885,871$ & $3,667,596$ \\
\hline 46 & Construcción & ICA SOC. CONT ROL. & -283064 & $-31,902$ & $-251,162$ & 5264065 & -225110 & $5,038,955$ \\
\hline 47 & Diversific ada & CORP.INTERAMER. & 749,731 & 83,021 & 666,710 & $2,692,347$ & $1,871,300$ & $4,563,647$ \\
\hline 48 & Diversific ada & ALFA & $5,324,651$ & $1,283,019$ & $4,041,632$ & $47,059,502$ & $7,192,681$ & $54,252,183$ \\
\hline 49 & Diversific ada & DESC & $2,321,475$ & 470,784 & $1,850,691$ & $15,662,013$ & $2,276,940$ & $17,938,953$ \\
\hline 50 & Diversific ada & GCARSO & $8,065,086$ & $2,937,902$ & $5,127,184$ & $35,375,067$ & $3,787,835$ & $39,162,902$ \\
\hline 51 & Diversific ada & GIND SALT IШO & 978,434 & 302,011 & 676,423 & $4,373,503$ & 849,323 & $5,222,826$ \\
\hline 52 & Diversific ada & GSANBORNS & $2,713,827$ & $1,069,911$ & $1,643,916$ & $14,063,478$ & $-190,693$ & $13,872,785$ \\
\hline 53 & Diversific ada & SANLUIS CORP. & 675,829 & 73,645 & 602,184 & $5,523,478$ & $-776,622$ & $4,746,856$ \\
\hline 54 & Diversific ada & CYDSA & 167849 & -79616 & 247,465 & 8045676 & -1833600 & $6,212,076$ \\
\hline 55 & Diversific ada & GACCIÓN & 197279 & 27870 & 169,409 & 3142700 & -154816 & $2,987,884$ \\
\hline 56 & Metalic os & CAMESA & 464781 & 236362 & 228,419 & 2682033 & 532195 & $3,214,228$ \\
\hline 57 & Metalic os & INDUSTRIAS CHB & 466066 & 77919 & 388,147 & 1809724 & 2747487 & $4,557,211$ \\
\hline 58 & Minera & GMEXICO & $5,522,949$ & $3,635,041$ & $1,887,908$ & $56,380,390$ & $3,410,589$ & $59,790,979$ \\
\hline 59 & Minera & PENOLES & 110,526 & $-64,740$ & 175,266 & $11,315,404$ & $2,031,043$ & $13,346,447$ \\
\hline 60 & Minerales no M & VIRO & $3,432,176$ & 977,002 & $2,455,174$ & $22,106,903$ & $-640,311$ & $21,466,592$ \\
\hline 61 & Quimica & TEKCHEM & 35671 & 2612 & 33,059 & 588426 & -42915 & 545,511 \\
\hline 62 & Quimica & MEXICHEM S.A. & 319271 & 130346 & 188,925 & 1376503 & 286297 & $1,662,800$ \\
\hline 63 & Serv. Financieros & BANAMEX & $10,335,350$ & $1,986,840$ & $8,348,510$ & $12,434,830$ & $86,631,000$ & $99,065,830$ \\
\hline 64 & Serv. Financieros & GFBITAL & 719080 & 342730 & 376,350 & 3785720 & 2731360 & $6,517,080$ \\
\hline 65 & Serv.Financieros & GFBBBVA BANCOMER & $4,891,600$ & 69,840 & $4,821,760$ & $16,156,911$ & $2,636,040$ & $18,792,951$ \\
\hline 66 & Serv.Financieros & GFINBURSA & $1,610,050$ & 927,170 & 682,880 & 245,080 & $23,584,280$ & $23,829,360$ \\
\hline 67 & Serv.Financieros & GF BANORTE & 643,450 & 158,460 & 484,990 & $3,136,630$ & $26,686,340$ & $29,822,970$ \\
\hline 68 & Servicios & AEROP. SURESTE & 391262 & 164,852 & 226,410 & 2347627 & 606263 & $2,953,890$ \\
\hline 69 & Servicios & MEDICA SUR & 105974 & 22,367 & 83,607 & 564993 & 98171 & 663,164 \\
\hline 70 & Siderúrgica & TAMSA & 967,917 & 443,278 & 524,639 & $7,109,019$ & $2,312,186$ & $9,421,205$ \\
\hline 71 & Siderúrgica & HYLSAMEX & 1284833 & 252359 & $1,032,474$ & 20079458 & 1056185 & $21,135,643$ \\
\hline & & & & & & & & \\
\hline & as: & & & & & & & \\
\hline & Dato tomado del A & uario Financiero 2000 public & r la Bols a Mexi & cana de Valores & & & & \\
\hline & Dato tomado del $\mathrm{A}$ & uario Financiero 2000 publice & or la Bols a Mexi & cana de Valores & & & & \\
\hline & La utilidad aiustad & es iqual a : La utilidad de op & $n$ menos los im & buestos & & & & \\
\hline & Dato tomado del $\mathrm{A}$ & uario Financiero 2000 public & or la Bols a Mexi & cana de Valores & & & & \\
\hline & Capital de T rabajo & calculado con los datos de los & tados financieros & activo circulante & asivo circulan & & & \\
\hline & EI capital invertido & siqual a: Activo Filo más Car & de Trabaio & & & & & \\
\hline & El Costo de Capita & o Tasa de Descuento se calcu & & & & & & \\
\hline & Costo de la Deu & :(Intereses pagados / Pasi & $n \cos t 0)-\mathrm{T} a \mathrm{a} a \mathrm{H}$ & scal & & & & \\
\hline & Costo del Capita & ropio: Cos to de la Deuda + & de ries $\mathrm{go}^{*}$ & & & & & \\
\hline & * Prima de ries $a$ & Costo de la deuda - Tasa re & bre de riesao) & & & & & \\
\hline & Eva del año es igu & Utilidad Aju & & & & & & \\
\hline
\end{tabular}




\begin{tabular}{|c|c|c|c|c|c|c|c|c|c|c|c|c|c|c|c|c|c|c|}
\hline \\
\hline & & & & & & & $\begin{array}{l}\text { CULO DEL CO } \\
\text { Añ }\end{array}$ & & APITAL A & & & & & & & & & \\
\hline & & & & & & & & & & & & & & & & & & \\
\hline & SECTOR & EMPRESA & CLAVE & IB & CAPITAL & PASIVO & SUMA & $\%$ & $\%$ & INTERES & & & & costo & COSTO & CPPC & \begin{tabular}{|l|} 
costo \\
\end{tabular} & CPPC \\
\hline & & & & & CONTABLE & CON COSTO & $\mathrm{CC}+\mathrm{P}$ & cc & PT & PAGADO & beta & if & $\mathrm{rm}$ & CC beta & PASIVO CC & beta & Cc Prima & Srima de $\mathbf{R}$ \\
\hline & Alimentos & BIMBO & BIMBO & 7.93 & $\begin{array}{r}15,576,759 \\
\end{array}$ & $4,377,298$ & $19,954,057$ & 0.7806 & 0.2194 & 391,2 & 0.48 & & nim & 0.089 & 0.0581 & 0.08 & \begin{tabular}{|l|l|}
0.103 \\
\end{tabular} & \\
\hline & Alimentos & CONTINENT & CONTAL & 7.31 & $4,949,275$ & $2,191,203$ & $7,140,478$ & 0.6931 & 0.3069 & 37,387 & 0.270 & 0.053 & \begin{tabular}{|l|}
0.129 \\
\end{tabular} & 0.0732 & 0.0111 & 0.0541 & 0.103 & 0.1030 \\
\hline & Alimentos & FEMSA & FEMSA & 5.43 & $17,000,023$ & $9,061,101$ & $26,061,124$ & 0.6523 & \begin{tabular}{|l|}
0.3477 \\
\end{tabular} & $1,039,323$ & 0.328 & 0.053 & \begin{tabular}{|l|}
0.129 \\
\end{tabular} & 0.0776 & 0.0746 & 0.0765 & 0.103 & 0.0931 \\
\hline & Alimentos & GMASECA & GRUMA & 7.15 & $9,072,874$ & $7,020,687$ & $16,093,561$ & \begin{tabular}{|l|}
0.5638 \\
\end{tabular} & 0.4362 & 798,433 & 0.170 & 0.053 & \begin{tabular}{|l|}
0.129 \\
\end{tabular} & 0.0655 & 0.0739 & 0.0692 & 0.103 & 0.0903 \\
\hline & Alimentos & GMODELO & GMODELO & 8.53 & $26,762,773$ & 267,000 & $27,029,773$ & 0.9901 & \begin{tabular}{|l|}
0.0099 \\
\end{tabular} & 3,278 & 0.540 & 0.053 & \begin{tabular}{|l|}
0.129 \\
\end{tabular} & 0.0938 & 0.0080 & 0.0930 & 0.103 & 0.1025 \\
\hline & Alimentos & SAVIA & SAVIA & 7.82 & $12,296,530$ & $12,073,265$ & $24,369,795$ & 0.5046 & 0.4954 & $1,359,722$ & 0.100 & 0.053 & \begin{tabular}{|l|}
0.129 \\
\end{tabular} & 0.0602 & 0.0732 & 0.0666 & \begin{tabular}{|c|}
0.103 \\
\end{tabular} & 0.0882 \\
\hline & Alimentos & BACHOCO & BACHOCO & 6.27 & $7,040,139$ & & $\begin{array}{l}, 808,525 \\
\end{array}$ & & 0.0984 & 135,084 & 0.170 & 0.053 & 0.129 & 0.0655 & & & & \\
\hline & $\begin{array}{l}\text { Alumentos } \\
\text { Alime }\end{array}$ & GEM UNIDAS & SGEUPECO & $\frac{6.27}{4.67}$ & $\begin{array}{r}1,, 040,159 \\
924,254\end{array}$ & $\frac{70,300}{114,315}$ & \begin{tabular}{|c|}
$, 000,, 025$ \\
$1,038,569$
\end{tabular} & $\frac{0.9010}{0.8899}$ & $\begin{array}{l}0.054 \\
0.1101\end{array}$ & $\frac{13,064}{24,101}$ & $\frac{.170}{0.328}$ & \begin{tabular}{|l|}
0.053 \\
0.053 \\
\end{tabular} & \begin{tabular}{|l|}
0.129 \\
0.129 \\
\end{tabular} & $\begin{array}{l}0.0050 \\
0.0776\end{array}$ & $\frac{0.143}{0.1370}$ & $\begin{array}{l}0.0841 \\
0.0841 \\
\end{array}$ & $\frac{0.170}{0.222}$ & $\begin{array}{l}0.1095 \\
0.2123 \\
\end{array}$ \\
\hline & Alimentos & GMODERNA & GMODERN & 6.19 & $1,680,493$ & 270,006 & $1,950,499$ & 0.8616 & 0.1384 & 52,500 & -0.040 & 0.053 & \begin{tabular}{|l|}
0.129 \\
\end{tabular} & 0.0495 & 0.1264 & 0.0601 & 0.200 & 0.1900 \\
\hline & Alimentos & ARGOS & ARSA & 6.9 & $2,532,751$ & 628,885 & $3,161,636$ & 0.8011 & 0.1989 & 145,655 & 0.110 & 0.053 & \begin{tabular}{|l|}
0.129 \\
\end{tabular} & 0.0609 & 0.1505 & 0.0788 & 0.249 & 0.2291 \\
\hline 11 & Alimentos & HERDEZ, S.A & AHERDEZ & 6.44 & $1,475,214$ & $1,079,992$ & $2,555,206$ & 0.5773 & $\begin{array}{l}.1 \\
0.4227 \\
\end{array}$ & $\begin{array}{l}14,003 \\
155,432 \\
\end{array}$ & 0.430 & \begin{tabular}{|l|}
0.053 \\
\end{tabular} & \begin{tabular}{|l|}
0.129 \\
\end{tabular} & 0.0854 & 0.0935 & 0.0888 & 0.135 & 0.1172 \\
\hline 12 & Alimentos & MAIZORO & MAIZORO & 4.45 & 236,280 & 97,105 & 333,385 & 0.7087 & 0.2913 & $\begin{array}{l}16,326 \\
\end{array}$ & 0.328 & \begin{tabular}{|l|}
0.053 \\
\end{tabular} & 0.129 & 0.0776 & 0.1093 & 0.0868 & 0.166 & 0.1495 \\
\hline 13 & Alimentos & KOF COCA & GKOFL & 5.79 & $6,482,356$ & $4,046,765$ & $10,529,121$ & 0.6157 & 0.3843 & 341,022 & 0.328 & 0.053 & \begin{tabular}{|l|}
0.129 \\
\end{tabular} & 0.0776 & 0.0548 & 0.0688 & 0.103 & 0.0845 \\
\hline & Alimentos & MASECA & MASECA & 6.92 & $5,067,984$ & 22,537 & $5,090,521$ & 0.9956 & 0.0044 & 24,313 & 0.410 & 0.053 & \begin{tabular}{|l|}
0.129 \\
\end{tabular} & 0.0839 & 0.7012 & 0.0866 & 1.350 & 1.3471 \\
\hline & Celulosa/Papel & KIMBERLY C & KIMBER & 8.53 & $11,481,500$ & $4,065,944$ & $15,547,444$ & 0.7385 & 0.2615 & 430,555 & 0.580 & 0.053 & \begin{tabular}{|l|}
0.129 \\
\end{tabular} & 0.0969 & 0.0688 & 0.0895 & 0.103 & 0.0941 \\
\hline 16 & Celulosa/Papel & EMPAQUES & IEMPAQ & 6.15 & $2,832,608$ & 723,572 & $3,556,180$ & 0.7965 & 0.2035 & 106,817 & -0.030 & 0.053 & \begin{tabular}{|l|}
0.129 \\
\end{tabular} & 0.0502 & 0.0960 & 0.0595 & 0.139 & 0.1306 \\
\hline 17 & Comercio & COM. MEXIC & COMERCI & 7.94 & $9,908,873$ & $1,749,269$ & \begin{tabular}{|l|}
$11,658,142$ \\
\end{tabular} & 0.8500 & $\begin{array}{l}0.1500 \\
0.15\end{array}$ & 237,952 & 0.740 & $\begin{array}{l}0.053 \\
0.053\end{array}$ & \begin{tabular}{|l|}
0.129 \\
0.129
\end{tabular} & 0.1091 & 0.0884 & 0.1060 & 0.124 & 0.1189 \\
\hline 18 & Come & SORIANA & SORIA & 8.22 & \begin{tabular}{|l|}
$9,707,982$ \\
9
\end{tabular} & 412,186 & $\mid \begin{array}{l}10,120,168 \\
\end{array}$ & 0.9593 & 0.0407 & 611 & 0.860 & \begin{tabular}{|l|}
0.053 \\
\end{tabular} & \begin{tabular}{|l|}
0.129 \\
\end{tabular} & 0.1183 & 0.0010 & 0.1135 & 0.103 & 0.1030 \\
\hline 19 & Comercio & ELEKTRA & \begin{tabular}{|l|} 
ELEKTRA \\
\end{tabular} & $\begin{array}{l}8.217 \\
8.17 \\
\end{array}$ & \begin{tabular}{|l|}
$5,297,150$ \\
5,297
\end{tabular} & $\begin{array}{r}4,374,679 \\
4,374\end{array}$ & \begin{tabular}{|c|}
$9,671,829$ \\
9,10
\end{tabular} & $\begin{array}{l}0.53477 \\
0.54\end{array}$ & $\begin{array}{l}0.4523 \\
0.45\end{array}$ & $\begin{array}{r}063,936 \\
\end{array}$ & 0 & \begin{tabular}{|l|}
0.053 \\
0.053 \\
\end{tabular} & \begin{tabular}{|l|}
0.129 \\
0.129 \\
\end{tabular} & $\begin{array}{l}0.11309 \\
0.1389\end{array}$ & 0.0986 & 0.1207 & $\begin{array}{l}0.103 \\
0.145\end{array}$ & 0.1239 \\
\hline 20 & Comercio & WALL MART & WALMEX & 8.28 & $31,091,726$ & & $31,091,726$ & 1.0000 & 0.0000 & & 1.040 & 0.053 & \begin{tabular}{|l|}
0.129 \\
\end{tabular} & 0.1321 & $\#$ \#DIV/O! & \#iDIV/0! & 0.103 & 0.1030 \\
\hline 21 & Comercio & SABA CASA & SAP & 5.54 & \begin{tabular}{|l|}
$2,339,398$ \\
\end{tabular} & $3,736,340$ & $\begin{array}{r}6,075,738 \\
\end{array}$ & 0.3850 & $\begin{array}{l}0.6150 \\
\end{array}$ & 208,553 & 0.621 & 0.053 & \begin{tabular}{|l|}
0.129 \\
\end{tabular} & 0.1000 & 0.0363 & 0.0608 & 0.103 & 0.0618 \\
\hline 22 & Comercio & DATAFLUX & DATAFLX & 6.57 & 623,016 & 408,578 & $1,031,594$ & 0.6039 & 0.3961 & 19,857 & 1.360 & 0.053 & \begin{tabular}{|l|}
0.129 \\
\end{tabular} & 0.1565 & 0.0316 & 0.1070 & 0.103 & 0.0744 \\
\hline 23 & Comercio & EDO & EDOARDO & 5.31 & 402 & 20,147 & 423,082 & 0.9524 & 0.0 & 5,484 & 0.621 & 0.053 & 0.129 & & & & 0.700 & 0.2954 \\
\hline 24 & Comercio & $\mathrm{ACO}$ & FRAGUA & 5.77 & 805,625 & 64,450 & 075 & 0.9259 & 0.0741 & 8,194 & 0.621 & 0. & 0.129 & 0.1000 & 326 & & 113 & $\frac{1105}{1105}$ \\
\hline 25 & Comercio & GCORVI & GCORVI & $\frac{5.71}{6.22}$ & \begin{tabular}{r|}
$1,198,265$ \\
\end{tabular} & $\begin{array}{r}4,400 \\
454,833 \\
\end{array}$ & \begin{tabular}{|r|}
$1,653,098$ \\
\end{tabular} & $\begin{array}{l}0.5259 \\
0.7249 \\
\end{array}$ & $\begin{array}{l}0.2751 \\
0.271\end{array}$ & $\begin{array}{l}0,154 \\
98,214\end{array}$ & \begin{tabular}{|c|}
0.221 \\
0.240
\end{tabular} & \begin{tabular}{|l|}
0.053 \\
0.053 \\
\end{tabular} & \begin{tabular}{|l|}
0.129 \\
0.129 \\
\end{tabular} & 0.0709 & $\begin{array}{l}0.0020 \\
0.1404\end{array}$ & 0.0900 & 0.173 & 0.2040 \\
\hline 26 & Comercio & GIGANTE & GIGANTE & 6.79 & $10,962,512$ & 678,313 & \begin{tabular}{|l|}
$11,640,825$ \\
\end{tabular} & 0.9417 & 0.0583 & 268,576 & 0.180 & 0.053 & \begin{tabular}{|l|}
0.129 \\
\end{tabular} & 0.0663 & 0.2574 & 0.0774 & 0.462 & 0.4503 \\
\hline 27 & Comercio & GPALACIO H & GPH & 6.22 & $\mid$ & $1,591,317$ & $5,985,281$ & 0.7341 & 0.2659 & 282,902 & 0.040 & 0.053 & \begin{tabular}{|l|}
0.129 \\
\end{tabular} & 0.0556 & 0.1156 & 0.0715 & 0.179 & 0.1618 \\
\hline$\frac{21}{28}$ & Comercio & ALSEA & ALSEA & 0.24 & 862,809 & 289.859 & $\mid$ & 0.7485 & 0.2515 & $\begin{array}{r}40,647 \\
40,647\end{array}$ & 0.021 & & \begin{tabular}{|l|}
0.129 \\
0.129 \\
\end{tabular} & 0.1000 & 11 & 0.0978 & 0.130 & 0.1201 \\
\hline 29 & Comercio & NADRO & NADRO & 5.8 & $2,011,562$ & 50,562 & $2,062,124$ & 0.9755 & \begin{tabular}{l|}
0.0245 \\
\end{tabular} & 5,164 & 0.621 & 0.053 & \begin{tabular}{|l|}
0.129 \\
\end{tabular} & 0.1000 & 664 & 0.0992 & 0.080 & 0.0799 \\
\hline 30 & Comercio & LIVERPOOL & LIVEPOL & 6.88 & 918 & 639,096 & $11,680,014$ & 0.7741 & 0.2259 & 31,772 & 0.000 & 0.05 & 0.129 & 0.0525 & & 535 & 0.103 & 0.09 \\
\hline 31 & Comercio & GPROVE QU & GPQB & & $\frac{1,040,10}{133,142}$ & $\frac{1009,050}{56,166}$ & $\mid 189,308$ & $\frac{0.1741}{0.7033}$ & $\begin{array}{l}0.2967 \\
0.2967\end{array}$ & $\frac{21,174}{16,434}$ & 0.602 & .05 & $\begin{array}{l}0.129 \\
0.129\end{array}$ & $\frac{0.0520}{0.1000}$ & 0.1902 & 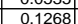 & $\frac{0.05}{0.328}$ & 0.28 \\
\hline 32 & Comunicaciones & TELECOM C & TELECOM & 9.19 & $21,957,447$ & $93,338,113$ & $115,295,560$ & 0.1904 & 0.8096 & $10,273,551$ & 0.621 & 005 & 0.129 & 0.1573 & $\frac{02}{15}$ & 80 & 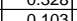 & 007 \\
\hline 33 & Comunic & TELEVISA & TLEVISA & 2.1 & 16,857,125 & 11,344,957 & $28,202,082$ & 0.5977 & \begin{tabular}{|l|}
0.4023 \\
\end{tabular} & 1,416,191 & 0.548 & \begin{tabular}{|l|l|}
0.053 \\
0.053
\end{tabular} & $\mid$\begin{tabular}{|l|}
$\mid 0.129$ \\
0.129 \\
\end{tabular} & $\begin{array}{l}0.153 / 3 \\
0.0945 \\
\end{array}$ & 311 & 91 & $\begin{array}{l}0.103 \\
0.110 \\
\end{array}$ & $\begin{array}{l}-0.07 / 5 \\
0.0982 \\
\end{array}$ \\
\hline 34 & Comunic & TELN & TELME & 7.08 & $50,006,400$ & $75,250,656$ & $125,257,056$ & 0.3992 & 0.6008 & $10,101,555$ & 0.920 & 0.053 & \begin{tabular}{|l|}
0.129 \\
\end{tabular} & 0.0429 & 0.0873 & 0.1015 & 0.122 & $\begin{array}{l}0.05011 \\
0.1011 \\
\end{array}$ \\
\hline 35 & Comunicaciones & TVAZTECA & TVAZTCA & & $4,013,740$ & $6,076,557$ & $10,090,297$ & 0.3978 & 0.6022 & 916,218 & 0.548 & 0.053 & \begin{tabular}{|l|}
0.129 \\
\end{tabular} & 0.0945 & 0980 & & 0.143 & 0.1161 \\
\hline 36 & Comunicaciones & TMM & TMM & 6.52 & 0,081 & $12,292,493$ & $13,792,574$ & 0.1088 & \begin{tabular}{l|}
0.8912 \\
\end{tabular} & 568,589 & -0.120 & 0.053 & \begin{tabular}{|l|}
0.129 \\
\end{tabular} & 0.0433 & 0.0301 & 0.0315 & 0.103 & 0.0379 \\
\hline & Comunicaciones & CINTRA & CINTRA & 6.31 & & $\begin{array}{l}5,606,072 \\
\end{array}$ & $12,317,139$ & 0.5449 & 0.4551 & 639,841 & 0.150 & & \begin{tabular}{|l|}
0.129 \\
\end{tabular} & & 42 & & 0.103 & 0.0899 \\
\hline & Comunicaciones & RADIO CENT & TRCENTRO & 6.45 & 33 & 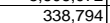 & $1,498,327$ & 0.7739 & & 17,000 & 0.260 & 0.053 & \begin{tabular}{|l|}
0.129 \\
\end{tabular} & 24 & 26 & & |03 & 871 \\
\hline & Comun & MOV & MOVILAB & & 64 & 4.802 & & 0.9870 & & 1,112 & 0.548 & 0.053 & \begin{tabular}{|l|}
0.129 \\
\end{tabular} & 0.0945 & & & 0.249 & 0.2473 \\
\hline 40 & Coms & IUSACELL & CELA & 6.57 & T. & $\begin{array}{ll}756,412 \\
\end{array}$ & 14,1 & 0.4520 & & $1,045,497$ & 0.710 & & \begin{tabular}{|l|}
0.129 \\
\end{tabular} & & & & & 0.1035 \\
\hline 41 & Constr & APAS & APASCC & & & 656 & 10,9 & 0.83 & 0.1 & 184 & 0.5 & 0.05 & 0.1 & & & & & $\begin{array}{l}0.0973 \\
0.053 \\
\end{array}$ \\
\hline 42 & Constr & CEME & $\frac{1 A C A S C B}{G C C B}$ & & & & & 0 & & 108 & 0. & & & & & & & \\
\hline 43 & Construcción & CEMEX, S.A. & CEMEX & 9.11 & & $\begin{array}{r}1,01,0<0 \\
56,329,445 \\
\end{array}$ & $\begin{array}{r}4,9 \\
1074\end{array}$ & $\begin{array}{l}0.0000 \\
0.4756 \\
\end{array}$ & & $\begin{array}{r}100,0050 \\
4541,772\end{array}$ & 0.450 & & & & & & & \\
\hline 44 & Constri & ARA & ARA & 7.47 & & 76,023 & $\frac{112}{112}$ & $\begin{array}{l}0.4700 \\
0.9709\end{array}$ & 0.0 & $\frac{4,401,712}{100,199}$ & 0.120 & \begin{tabular}{|l|}
0.053 \\
0.053
\end{tabular} & \begin{tabular}{|l|}
0.129 \\
0.129 \\
\end{tabular} & & 6 & & $\mid \begin{array}{l}\mid .1051 \\
1.661\end{array}$ & $\begin{array}{l}0.0 / 62 \\
1.6375\end{array}$ \\
\hline 45 & 5 Cons & GES & GEO & 7.47 & & & & 0.4817 & & 190,429 & 0.830 & 0.053 & \begin{tabular}{|l|l|}
0.129 \\
\end{tabular} & & & & 0.103 & 0.0775 \\
\hline 46 & Cons & $\mathrm{CO}$ & DICA & 6.91 & & & 16,6 & 0.5 & 0. & 1,311 & 0.390 & 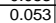 & \begin{tabular}{|l|}
0. \\
\end{tabular} & & & & & 0.1457 \\
\hline 47 & Divers & CORP.INTER & AIEB & 8 & & 2 & 7,4 & 0.6143 & & 74 & 0.8 & 0.0 & & & & & & .0923 \\
\hline 48 & Divers & ALF & ALF & 8.81 & & & & 0.3 & & 3,48 & & & & & & & & 310 \\
\hline 49 & Divers & DES & DES & 7.43 & & 12,2 & 21,4 & $\begin{array}{l}0.4282 \\
\end{array}$ & & 1,16 & 0.590 & & \begin{tabular}{|l|}
0.129 \\
\end{tabular} & & & & & 0.0794 \\
\hline 50 & Diver & $\mathrm{GC}$ & $\mathrm{GC}$ & 8.66 & & & $51,$. & $\begin{array}{l}0.420 \mathrm{~L} \\
0.4492 \\
\end{array}$ & & & & 0.0 & & & & & & 0.1250 \\
\hline 51 & Dive & SALTIL & GISSAB & 7.24 & & & & 0.6 & & 108 & 0.3 & & 0. & & & & & 0796 \\
\hline 52 & Diver & & SGSANBC & 7.76 & & & & 0.45 & & 2,28 & 0.840 & 0.0 & & & & & & \\
\hline 53 & Diver & & & & & & & & & $\frac{205}{605}$ & 0.120 & & & & & & & \\
\hline 54 & Divers & & & & & 5,6 & & 0.4 & & 821,718 & 0.669 & & 0. & & & & & 0.1129 \\
\hline 55 & Diversificada & & GACCION & 5.19 & 422 & $2,123,960$ & 3,42 & 0.3799 & 0.6 & 199,297 & $\begin{array}{l}0.0609 \\
0.669\end{array}$ & \begin{tabular}{|l|}
0.053 \\
\end{tabular} & \begin{tabular}{|l|}
0.129 \\
\end{tabular} & 0.1036 & 0.0610 & 0.0772 & 0.103 & 0.0770 \\
\hline 56 & Metal & tin & CAMES & 5.7 & & & & 0.74 & 0.8 & 98,939 & 0.130 & & \begin{tabular}{|l|}
0.129 \\
\end{tabular} & & & & & .1563 \\
\hline 57 & Metal & & $\mathrm{IICH}$ & 6.31 & & & & & & & & & & & & & & \\
\hline 58 & Mine & & $G N$ & 8. & & 27,4 & & 0.4 & & $2,691,933$ & & & 0.1 & & & & & 27 \\
\hline 59 & Mine & & PE\&OLES & & & & & 0.6 & & 325,329 & 0.130 & & & & & & & \\
\hline 60 & Minerales no $\mathrm{M}$ & VITRO & VITRO & 7.02 & $6,163,920$ & $15,879,697$ & $22,043,617$ & 0.2796 & 0.7204 & $2,158,350$ & 0.320 & 0.053 & \begin{tabular}{|l|}
0.129 \\
\end{tabular} & & 0.0883 & 0.0852 & 0.124 & 0.0984 \\
\hline 61 & Quimica & AEM & TEKCHE & 4.18 & 371,409 & 138,312 & 509,721 & $\begin{array}{l}0.7287 \\
0.728\end{array}$ & $\begin{array}{l}.2713 \\
0.274\end{array}$ & 20,682 & $\begin{array}{r}0.0<0 \\
0.669 \\
\end{array}$ & 0.053 & \begin{tabular}{|l|}
0.129 \\
0.129
\end{tabular} & & $\begin{array}{l}0.0972 \\
0.097\end{array}$ & & $\begin{array}{l}0.144 \\
0.142\end{array}$ & 0.1298 \\
\hline 62 & Quimi & EM S & SMEXCH & & 120 & & 1,37 & 0.8944 & & 9,256 & 0.669 & & 0.129 & & & & & . \\
\hline 63 & Serv. Fin & & & & & 71 & & & & 812 & & & & & & & & \\
\hline 64 & Serv. Fir & & & 2 & & & & & & 19,187 & & & 0.1 & & & & & 209 \\
\hline 65 & Serv.Financieros & GFB & AGFBB & 6.83 & $31,959,260$ & $349,459,390$ & 381,4 & 0.0838 & 0.9 & $53,475,850$ & 0.760 & 0.053 & \begin{tabular}{|l|}
0.129 \\
\end{tabular} & 0.1106 & 0.0995 & 0.1004 & $\begin{array}{ll}0.146 \\
\end{array}$ & 0.1034 \\
\hline 66 & Serv.Financieros & JRSA & GFINBUR & 772 & $18,943,060$ & $22,483,180$ & $41,426,240$ & 0.4573 & 0.5427 & $5,766,860$ & 0.390 & 0.053 & \begin{tabular}{|l|}
0.129 \\
\end{tabular} & 0.0823 & 0.1667 & 0.1281 & 0.281 & 0.2189 \\
\hline 67 & Serv.Financieros & & GFNORTE & 7.93 & & 91,02 & $96,584,240$ & 0.0576 & 0.9424 & $15,992,300$ & 0.737 & 0.053 & \begin{tabular}{|l|}
0.129 \\
\end{tabular} & 0.1089 & 0.1142 & 0.1139 & 0.176 & 0.1178 \\
\hline 68 & Servic & & RASU & 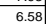 & & & 10,3 & 0.0719 & & & 0.220 & & & & & & & \\
\hline 69 & Servicios & SUR & AME & & & & & 0.7 & & 12 & 0.220 & & 0.129 & & & & 03 & \\
\hline 70 & Siderúrgica & TAMSA & TAMSA & 7.34 & \begin{tabular}{|l|}
$7,106,987$ \\
\end{tabular} & $1,305,810$ & $8,412,797$ & 0.8448 & 0.1552 & 135,833 & 0.290 & 0.053 & \begin{tabular}{|l|}
0.129 \\
\end{tabular} & 0.0747 & 0.0676 & 0.0736 & 0.103 & 0.0975 \\
\hline 71 & Siderúrgica & HYLSAMEX & HYLSAMX & 6.97 & $9,374,441$ & 14,061,493 & $23,435,934$ & 0.4000 & 0.6000 & $1,644,105$ & 0.670 & 0.053 & \begin{tabular}{|l|}
0.129 \\
\end{tabular} & 0.1038 & 0.0760 & 0.0871 & 0.103 & 0.0868 \\
\hline
\end{tabular}


